\title{
Radiomics in esophageal and gastric cancer
}

\author{
Bert-Ram Sah, ${ }^{1}$ Kasia Owczarczyk, ${ }^{1}$ Musib Siddique, ${ }^{1}$ Gary J. R. Cook,,${ }^{1,2}$ and \\ Vicky Goh (1) 1,3,4 \\ ${ }^{1}$ Department of Cancer Imaging, School of Biomedical Engineering and Imaging Sciences, King's College London, London, UK \\ ${ }^{2}$ King's College London and Guy's and St Thomas' PET Centre, St Thomas' Hospital, London, UK \\ ${ }^{3}$ Department of Radiology, Guy's St Thomas' Hospitals NHS Foundation Trust, London, UK \\ ${ }^{4}$ Radiology, Level 1, Lambeth Wing, St Thomas' Hospital, Westminster Bridge Road, London SE1 7EH, UK
}

\section{Abstract}

Esophageal, esophago-gastric, and gastric cancers are major causes of cancer morbidity and cancer death. For patients with potentially resectable disease, multimodality treatment is recommended as it provides the best chance of survival. However, quality of life may be adversely affected by therapy, and with a wide variation in outcome despite multi-modality therapy, there is a clear need to improve patient stratification. Radiomic approaches provide an opportunity to improve tumor phenotyping. In this review we assess the evidence to date and discuss how these approaches could improve outcome in esophageal, esophago-gastric, and gastric cancer.

Key words: Esophageal cancer-Esophagogastric junction cancer-Radiomics-Computed tomography-Magnetic resonance imaging - Positron emission tomography

\section{The need for better patient stratification}

Esophageal or esophago-gastric cancer $(456,000$ new cases annually) and gastric cancer (952,000 new cases annually) are leading causes of cancer deaths worldwide [1]. Above $50 \%$ of presenting patients are diagnosed with stage IV disease, precluding curative treatment. For patients with early stage disease, surgery, often combined with neoadjuvant chemotherapy or chemoradiotherapy, offers the best chance of cure [2-7].

As an example, data from the OEO2 and MAGIC trials for esophageal and esophago-gastric cancer have shown a 6\% [3] and 13\% [4] improvement in 5-year overall-survival, respectively. Trimodality therapy for

Correspondence to: Vicky Goh; email: vicky.goh@kcl.ac.uk esophageal and esophago-gastric cancer combining neoadjuvant chemo- and radiation-therapy in addition to surgery may also be superior to neoadjuvant chemotherapy alone in a selected patient population [8]. The CROSS trial [6] comparing neoadjuvant chemoradiotherapy plus surgery with surgery alone in patients with esophageal and esophago-gastric cancer showed a superior overall-survival of 49 vs. 24 months, hazard ratio $0.657, \mathrm{p}=0.003$, and a pathological complete response rate of $29 \%$, for patients with multi-modality treatment with no increase in surgical mortality (4\% in surgery and trimodality groups, respectively).

A recent systematic review and meta-analysis of neoadjuvant chemotherapy in patients with gastric cancer has also found improved 3-year survival rates (relative risk 1.30; 95\% CI 1.06-1.59, $p<0.01$ ) [9]. Typical management pathways are shown in Figs. 1 and 2 for esophageal, esophago-gastric, and gastric cancer, respectively. Nevertheless overall-survival remains poor despite these improvements in patient care.

Recent genomic analyses have highlighted the genetic heterogeneity present in esophageal, esophago-gastric [10], and gastric cancer [11, 12] as an underlying cause for the differences in outcome and heterogeneity of response to therapy. Quality of life also remains poor for many patients post-surgery, taking up to 3 years to return to pre-therapy levels in patients undergoing esophageal resection [13]. Better patient stratification remains a key challenge for patients with upper gastrointestinal tract cancers.

\section{The imaging pathway at staging}

For esophageal and esophago-gastric cancer, contrastenhanced computed tomography (CT) remains the most commonly performed first step in staging due to the high prevalence of metastatic disease at presentation [14-16]. For patients being considered for a curative pathway, endoscopic ultrasound (EUS) and 18F-fluorodeoxyglu- 
Diagnosis: Endoscopy

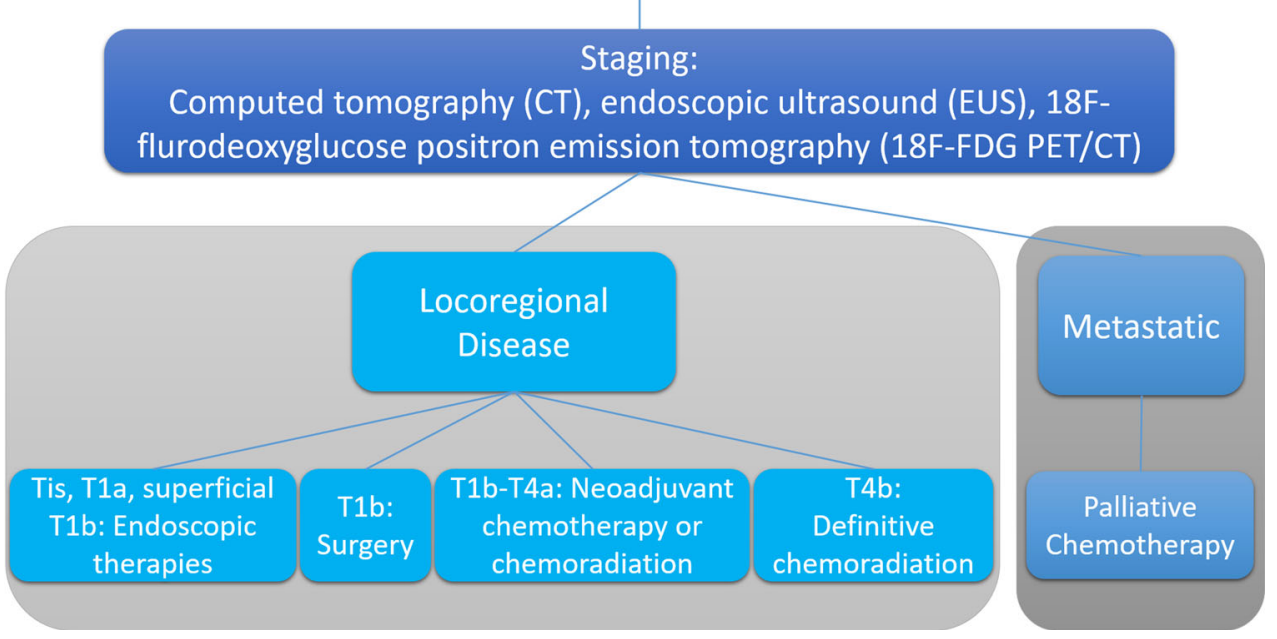

Fig. 1. Typical pathways for the management of patients with newly diagnosed esophageal and esophago-gastric cancer.

Diagnosis: Endoscopy

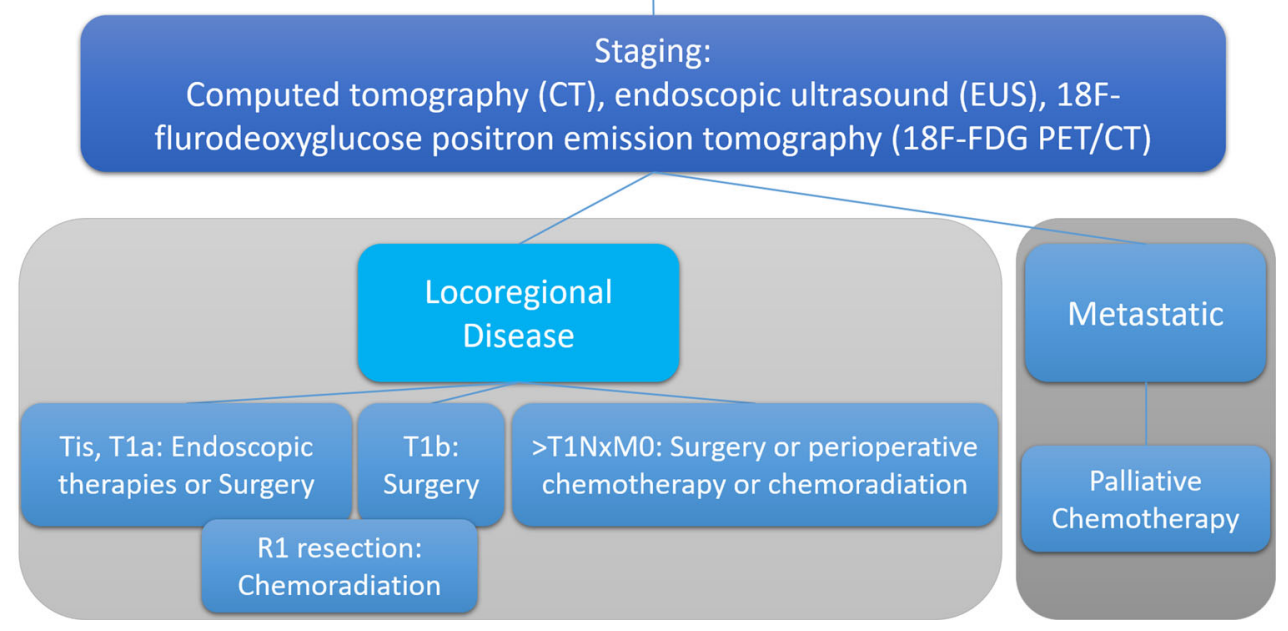

Fig. 2. Typical pathways for the management of patients with newly diagnosed gastric cancer.

cose positron emission tomography/CT (18F-FDG PET/ CT) are performed due to the high sensitivity and specificity of EUS for local tumor and nodal staging; and 18F-FDG PET for distant metastases [17-19]. This aims to reduce the futile surgery rate.

For gastric cancer, initial staging is again by contrastenhanced CT. If curative treatment is being considered, the use of endoscopic ultrasound (EUS) is helpful in determining the proximal and distal extent of the tumor, whereas 18F-FDG PET/CT has been shown to improve staging by detecting involved lymph nodes and metastatic disease, although it can be less accurate in mucinous and diffuse tumors [20].

In esophageal cancer, PET has the potential to change management in up to a third of patients [21, 22], and is often incorporated into radiotherapy planning pathway
[23, 24]. The American College of Surgeons Oncology Group reported sensitivity and specificity of 18 F-FDGPET/CT scans to be $79 \%$ and $95 \%$, respectively [18].

Magnetic resonance imaging (MRI) is currently not recommended for the routine imaging of esophageal or gastric cancer. However, with the recent advent of hybrid PET/MRI systems in clinical practice, there has been growing interest in MRI's ability as an assessment tool. MRI provides excellent soft-tissue contrast, and may demonstrate the esophageal wall layers and adjacent nodes. Physiological sequences (e.g., diffusion-weighted MRI) may also be included as part of the protocol. An initial staging $18 \mathrm{~F}-\mathrm{FDG}$ PET/MRI study with a pathology gold standard has been promising for nodal assessment with reported accuracy of $83 \%$ compared to $75 \%$ and $50 \%$ for EUS and CT, respectively [25]. 


\section{A role for radiomics?}

Radiomic approaches are showing promise for patient stratification. Radiomics exploit the data performed as part of the clinical management pathway. In terms of imaging, a number of parameters may be extracted and combined including standard descriptors (e.g., size, morphology, TNM (tumor, node, metastasis) stage); qualitative, semi-quantitative, or quantitative physiological parameters (e.g., contrast enhancement, diffusion characteristics, tracer uptake); and additional agnostic features which are otherwise 'invisible', with bioinformatic approaches. Of these, texture-based features have been investigated most commonly to date. Table 1 highlights some features that have been investigated in studies.

Radiomic signatures provide additional information predictive of underlying tumor biology and behavior. These signatures can be used alone or with other patientrelated data (e.g., pathological data; genomic data) to improve tumor phenotyping, treatment response prediction and prognosis. Radiomic signatures may be obtained for all cross-sectional imaging modalities, including CT, PET, and MRI. Figure 3 illustrates a typical radiomics pipeline.Figure 4 demonstrates the process of tumor segmentation for a 18F-FDG PET image with a corresponding plot of standardized uptake value for the tumor. Initial studies in esophageal, esophago-gastric, and gastric cancer have shown promise for patient care.

\section{F-FDG PET radiomics}

Nine 18F-FDG PET studies have been performed in esophageal and esophago-gastric cancer and are summarized in Table 2. As yet no studies have been performed for gastric cancer. Studies to date have focused on the prediction of response or prognosis in comparison to standard practice. Studies have found that various first, second and high-order features have been contributory to the assessment of response, differentiating be-

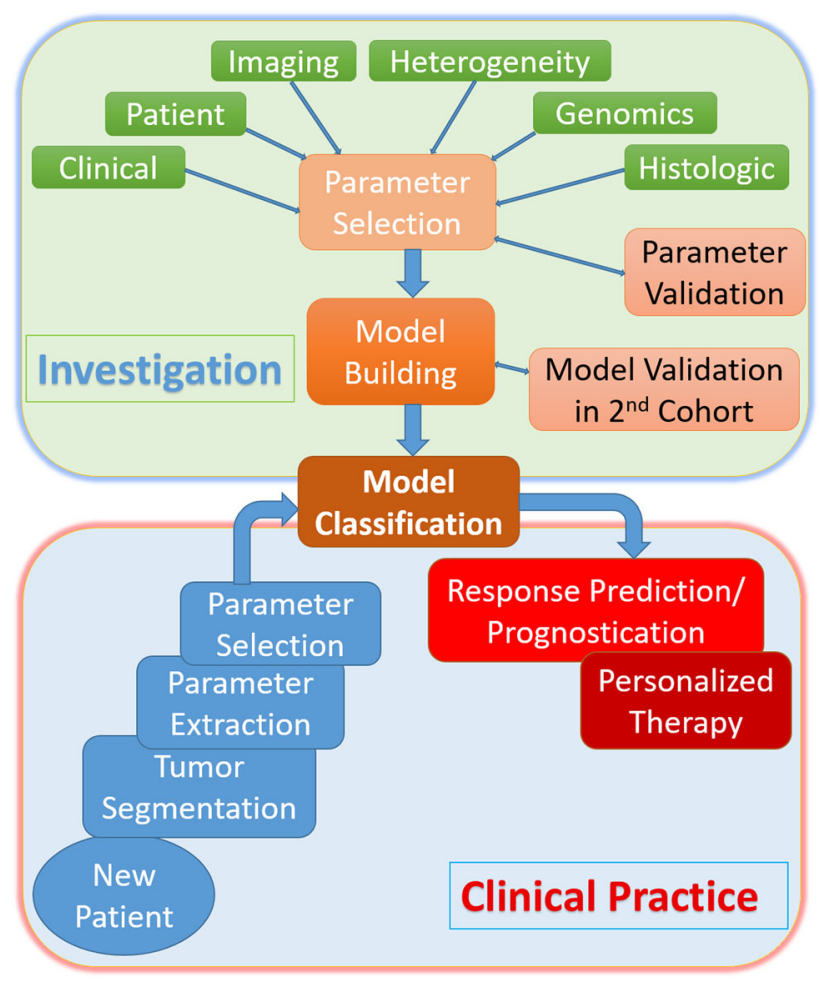

Fig. 3. Schema demonstrating typical radiomics pipeline.

tween responders and non-responders (with greater heterogeneity in non-responders), as well as being predictive of complete response. Performance has been better than conventional parameters alone. Prognostication data remain conflicting.

In greater detail, five studies have investigated the prediction of response to therapy alone $(n=2)$; prognosis alone $(n=1)$ and the prediction of response to therapy and prognosis $(n=2)$ from pre-therapy imaging. One of the earliest studies by Tixier et al. showed in 41 patients that gray-level co-occurrence matrix (GLCM) homogeneity, GLCM entropy, gray-level size-zone matrix (GLSZM) size-zone variability and run length matrix (RLM) intensity variability differentiated non-respon-

Table 1. Overview of features used in radiomics

Feature-group

First-order-histogram statistics

Second-order gray-level co-occurrence matrix (GLCM) statistics

Second-order gray-level difference matrix (GLDM) statistics

High-order neighborhood gray-tone difference matrix (NGTDM) statistics

High-order gray-level run-length (GLRL or RLM) statistics

High-order gray-level size zone matrix (GLSZM) statistics

Fractal analysis
Parameter examples

Mean, median, skewness, kurtosis, energy (uniformity), entropy

Entropy, homogeneity, energy (uniformity), contrast, autocorrelation, cluster shade, cluster prominence, difference entropy, difference variance, dissimilarity, inverse difference moment, maximum probability, sum average, sum entropy, sum variance

Mean, entropy, variance, contrast

Coarseness, contrast, busyness, complexity, texture strength

Short run emphasis, long run emphasis, gray-level nonuniformity, run-length nonuniformity, intensity variability, run-length variability

Short-zone emphasis, long-zone emphasis, intensity nonuniformity, zone percentage, intensity variability, size zone variability

Mean fractal dimension, standard deviation, lacunarity, Hurst component 

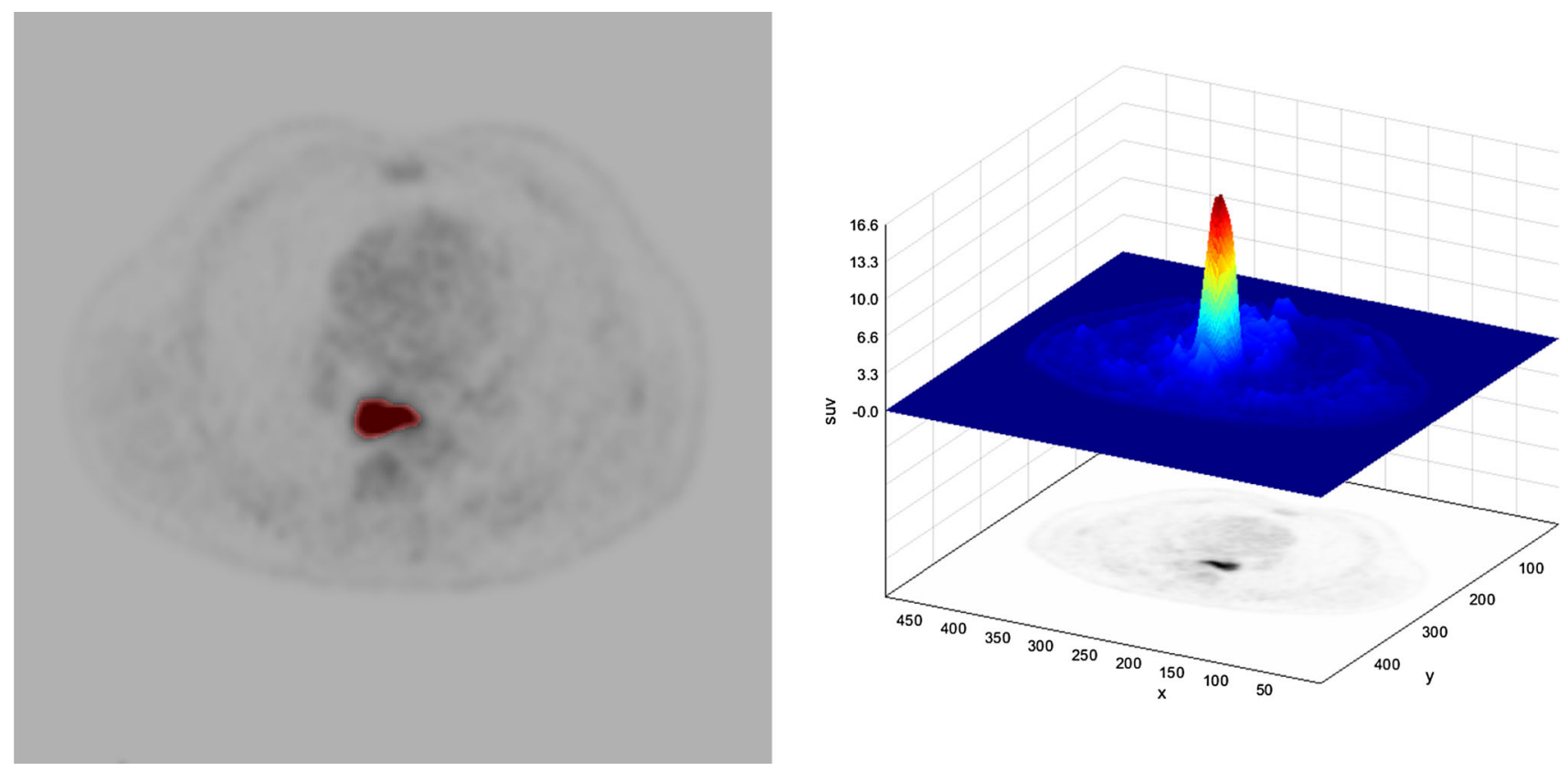

Fig. 4. Example of tumor segmentation for extraction of radiomic features from an axial PET image. In the right image the corresponding standardized uptake values for the region-of-interest is displayed.

ders, partial-responders, and complete-responders with sensitivities of $76 \%-92 \%$ [26]. Beukinga et al. showed in 97 patients that a clinical model including PET-derived gray-level run length (GLRL) long run low gray level emphasis and CT-derived run percentage had a higher area under the receiver operator curve (AUROC) compared to maximum standardized uptake value $\left(\mathrm{SUV}_{\max }\right)$ in predicting therapy response [27].

In a study of 52 patients with squamous cancers, Nakajo et al. found that 18F-FDG PET/CT GLSZM intensity variability, and GLSZM size-zone variability, as well as standard volumetric parameters, such as metabolic tumor volume (MTV) and total lesion glycolysis (TLG), were predictors of tumor response but not of progression free or overall-survival [28]. Non-responders showed significantly higher intensity variability and sizezone variability. Similarly, in a study of 65 patients Paul et al. found that a model incorporating GLCM homogeneity was a predictor of response (with an AUROC value of 0.823) but not of survival [29]. However, in a larger study with 403 patients, Foley et al. found that total lesion glycolysis, histogram energy and kurtosis were independently associated with overall-survival [30].

Four studies have assessed pre- and post-therapy 18F-FDG PET imaging. An initial study by Tan et al. found in 20 patients that $2 \mathrm{SUV}_{\text {mean }}$ parameters, $\mathrm{SUV}_{\text {mean }}$ decline and $\mathrm{SUV}_{\text {mean }}$ skewness, and 3 texture features GLCM inertia, GLCM correlation, and GLCM cluster prominence, were significant predictors of complete response with an AUROC of 0.76 [31]. In 217 patients with adenocarcinoma, Van Rossum et al. developed a prediction model which included change in run length matrix (RLM) run percentage, change in GLCM entropy, and post-chemoradiation roundness, and increased the corrected c-index (concordance-index, comparable to AUROC) from 0.67 to 0.77 , compared to the clinical model alone [32]. Yip et al. found that a change in run length and size-zone matrix differentiated responders from non-responders [33]. More recently, Beukinga et al. found that clinical T-staging combined with post-chemoradiotherapy ${ }^{18} \mathrm{~F}$-FDG PET orderliness provided high discriminatory accuracy in predicting pathologic complete response compared to clinical variables or SUVmax alone [34].

\section{CT radiomics}

Nine studies have investigated the ability of CT-derived heterogeneity parameters for classification, prediction of response and overall-survival in patients with esophageal or gastric cancer. Three studies have been performed for esophageal cancer in terms of prediction of response or prognosis (Table 3). These have found that greater heterogeneity is present in non-responders and those with poorer outcome.

The largest study in 49 patients found that histogram skewness, histogram kurtosis, GLSZM long-zone emphasis, and 2 Gabor transformed parameters MSA-54 and MSE-54, discriminated non-responders from responders using an artificial neural network-derived prediction model [35]. The two remaining studies have assessed prognostication. Ganeshan et al. found that lower histogram uniformity (with Gaussian filtration) from unenhanced $\mathrm{CT}$ images before start of treatment 


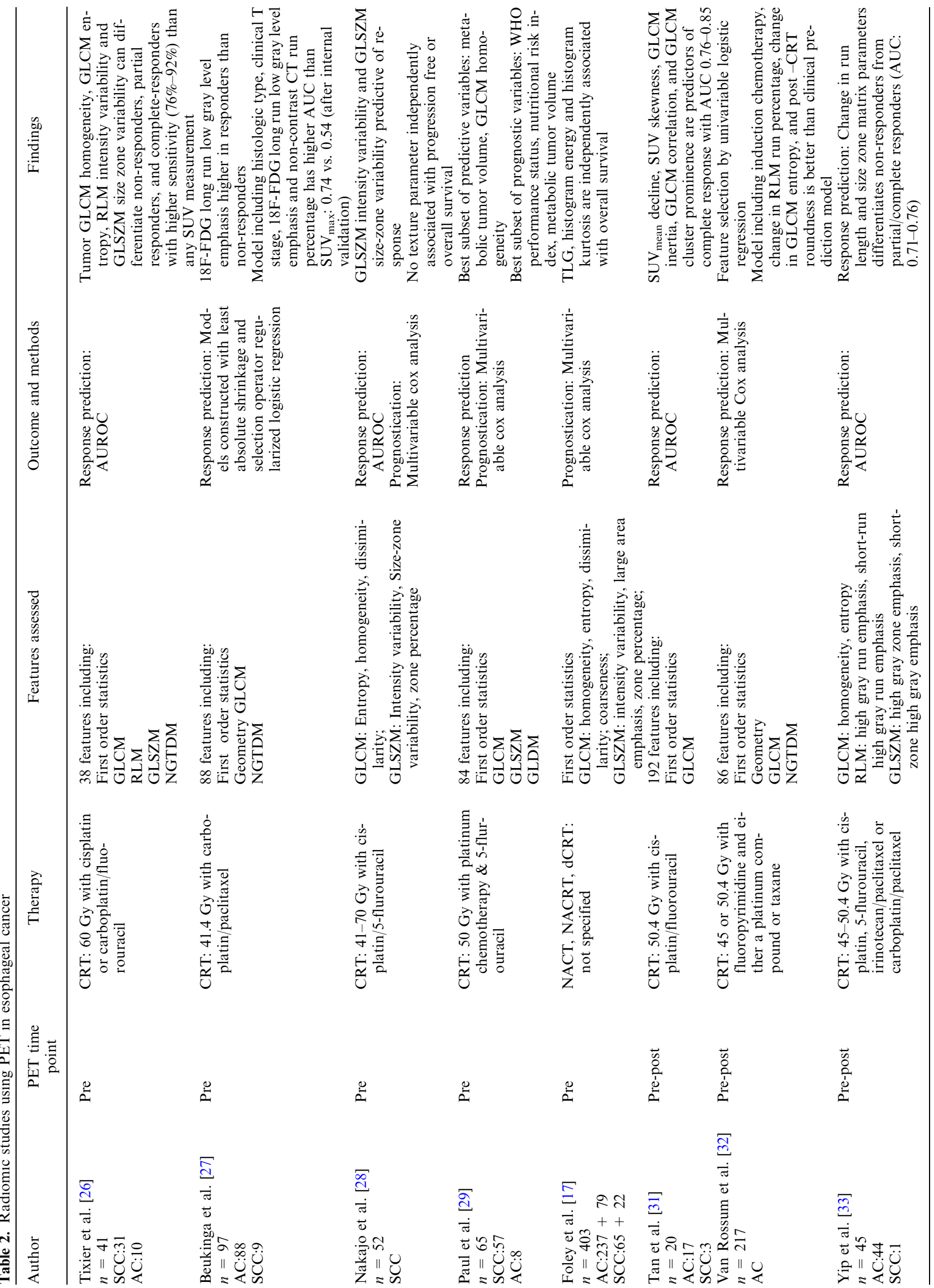



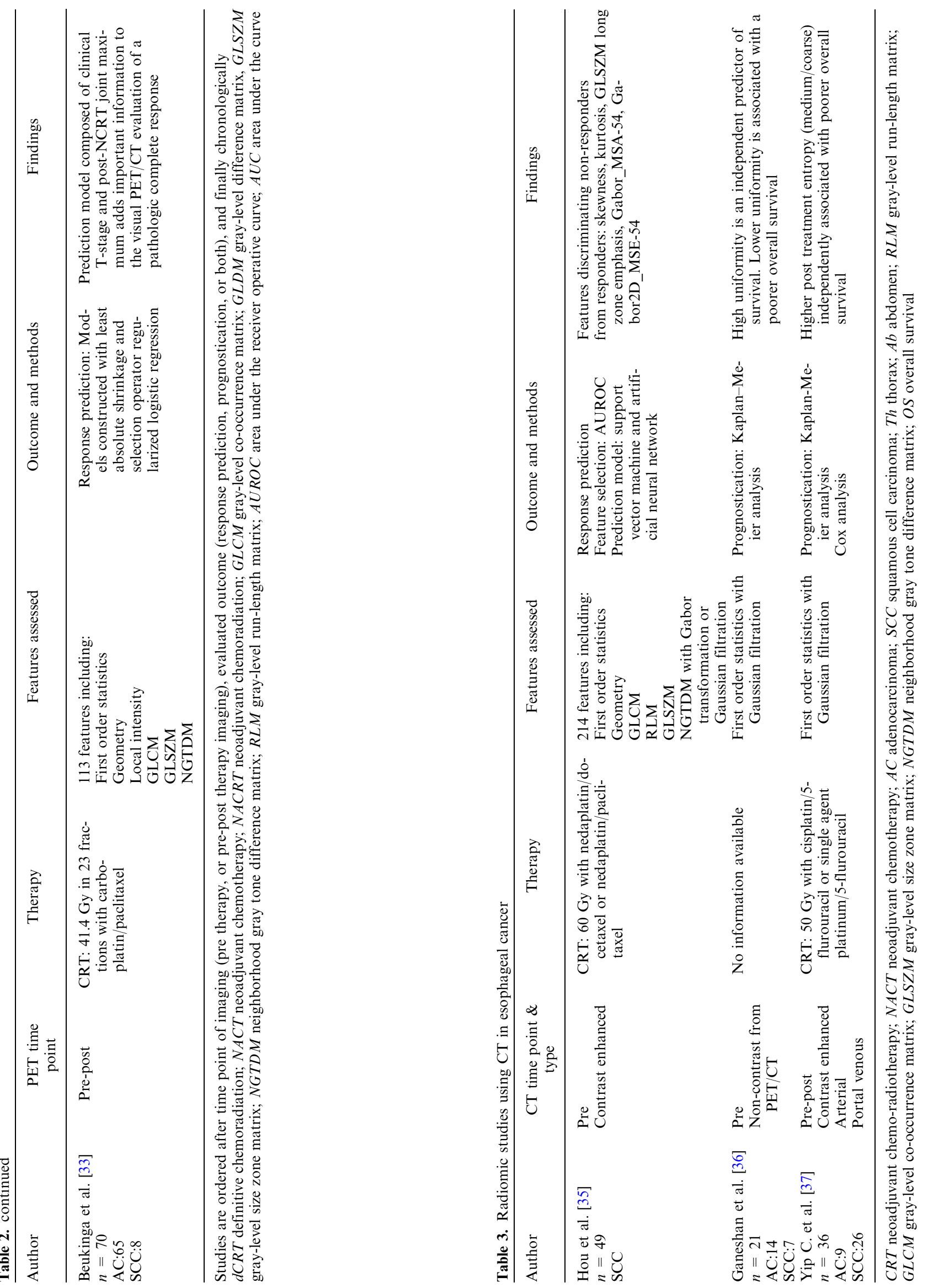


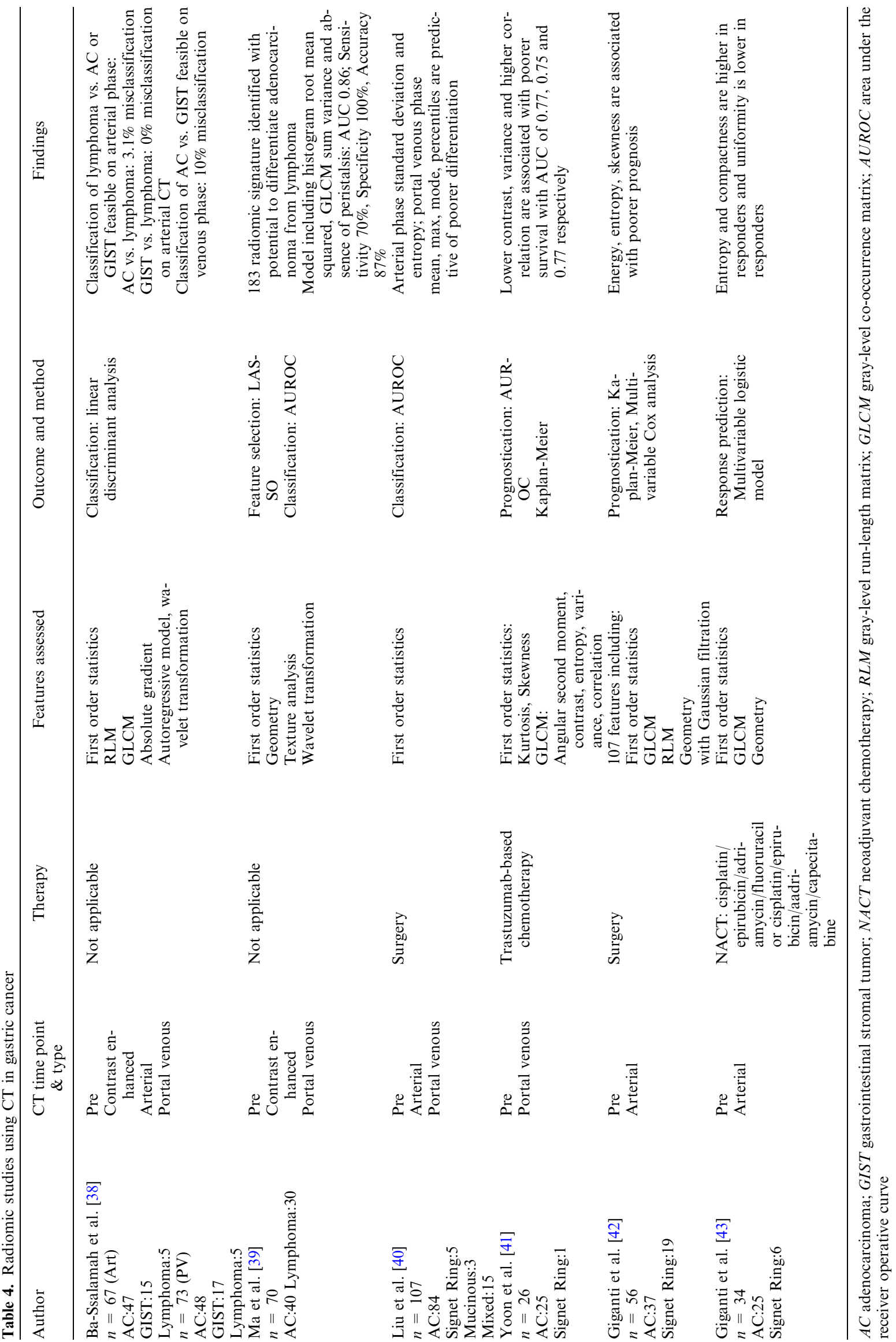




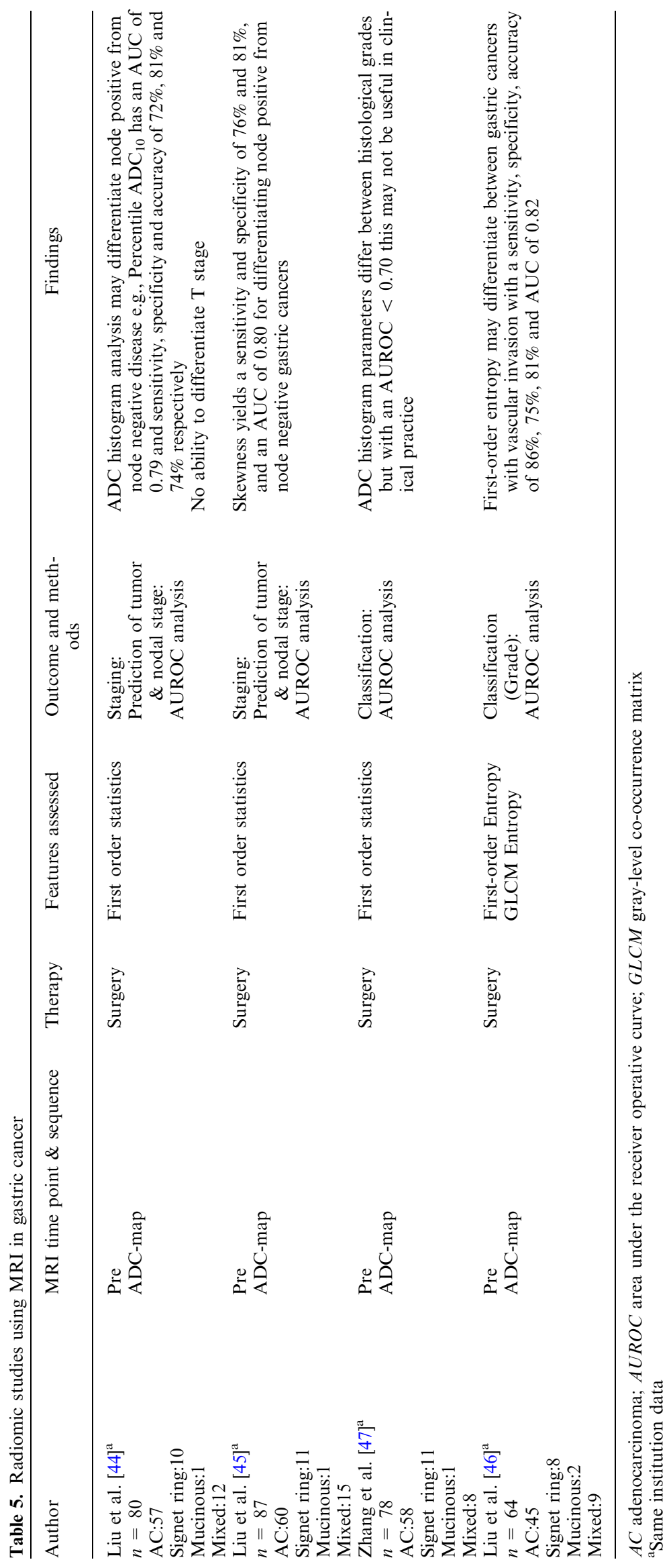


was an independent predictor for poorer overall-survival [36].Yip et al. analyzed contrast-enhanced images of 36 patients before and after treatment and found a significant decrease in histogram entropy and increase in uniformity (with Gaussian filtration) between the two time points. Higher post treatment entropy was associated with poorer overall-survival [37].

For gastric cancer (Table 4), three studies have assessed the potential of radiomic approaches for classification. Studies have found that first and second-order analysis in the contrast-enhanced images may help in differentiation of lymphoma from gastrointestinal stromal cancer [38] or adenocarcinoma [39]. Another study in 107 patients found that arterial phase standard deviation and entropy were correlated with poorer differentiation [40]. For prognostication, Yoon et al. investigated 26 HER 2 + gastric cancer patients before trastuzumabtreatment. In their analysis, they found GLCM contrast, variance, correlation and angular second moment (also known as energy or uniformity) were associated with a poorer survival [41]. In another study, Giganti et al. showed in 56 patients, that first-order energy, entropy, and skewness were significantly associated with a negative prognosis [42]. Giganti et al. also assessed pre chemotherapy texture features derived from the late arterial phase of 34 patients. They found entropy and compactness were higher and uniformity lower in responders [43]. No studies have assessed prognostication or response to therapy in gastric cancer.

\section{MRI radiomics}

To date there have been little data for MRI in this tumor group as MRI is not performed routinely in the clinical pathway. There have been some exploratory data of pretherapeutic ADC-maps of gastric cancer (Table 5). Liu et al. found that first-order statistics skewness may differ from node positive to node negative patients, and are associated with pathological characteristics including perineural and vascular invasion [44-47]. However, no studies so far have investigated prognostication or response assessment.

\section{Discussion}

To date 22 imaging studies have been published investigating radiomic approaches in esophageal, esophagogastric, and gastric cancer, predominantly focused on texture analysis. Preliminary data for esophageal and esophago-gastric cancer suggest that there is potential for radiomic approaches in improving patient stratification for therapy. Eight 18F-FDG PET studies investigated the feasibility of heterogeneity analysis for response prediction (four studies with pre-therapy imaging only). Among the most often reported significant feature was GLCM entropy, reflecting the local randomness (irregularity) within the image, and where low GLCM entropy represents a more homogeneous texture. The reported accuracy for successful classification of therapy response ranged from 0.7 to 1.0 (AUC).

Nearly all published studies incorporated "classical" PET parameters e.g., $\mathrm{SUV}_{\max }$, total lesion glycolysis and metabolic tumor volume into predictive models. In general radiomic parameters contributed to predictive models and provided additional information to standard parameters. Three CT studies of esophageal cancer have also suggested that greater tumoral heterogeneity is associated with poor response and outcome.

PET-studies investigating texture features as a prognosticator were more mixed. Only two studies found associations with overall-survival. The CT and MRI data for gastric cancer were also varied. Two studies found several features to be associated with survival time, however, for some parameters, e.g., histogram entropy and energy, it was surprising to find both parameters to be associated in the same direction given what they represent mathematically.

A challenge for interpretation of studies to date is the use of retrospective datasets with different imaging techniques across different scanners and/or institutions; different methodologies for feature selection; the focus on different feature sets; the lack of transparency in methodology with the use of different in-house software; as well as varying statistical and bioinformatics approaches for data analysis and interpretation. This has been highlighted by researchers in the field [48].

Moving forward in the context of esophageal and esophago-gastric cancer, it is important to improve our data quality. Planned prospective studies incorporating quality control is a step in the right direction to improving data curation and ensuring prediction models are fit for purpose and fulfill the promise of radiomics for improving patient stratification.

Acknowledgements The authors acknowledge support from the
Department of Health via the National Institute for Health Research
Comprehensive Biomedical Research Centre award to Guy's \& St
Thomas' NHS Foundation Trust in partnership with King's College
London and King's College Hospital NHS Foundation Trust; from the
King's College London/University College London Comprehensive
Cancer Imaging Centre funded by Cancer Research UK and Engi-
neering and Physical Sciences Research Council (EPSRC) in association
with the Medical Research Council and Department of Health [(C1519/
A16463)]; and Wellcome EPSRC Centre for Medical Engineering at
King's College London (WT 203148/Z/16/Z). Bert-Ram Sah received a
research Grant from the Swiss National Science Foundation.

Compliance with Ethical Standards

Conflict of interest Bert-Ram Sah, Kasia Owczarczyk, Musib Siddique and Gary Cook declare that he/she have no conflicts of interest. Vicky Goh receives research support from Siemens Healthcare; this is not related to the topic of this manuscript.

Human and Animal Participants This review article does not contain any studies with human participants or animals performed by any of the authors. 
Open Access This article is distributed under the terms of the Creative Commons Attribution 4.0 International License (http://creativecom mons.org/licenses/by/4.0/), which permits unrestricted use, distribution, and reproduction in any medium, provided you give appropriate credit to the original author(s) and the source, provide a link to the Creative Commons license, and indicate if changes were made.

\section{References}

1. Globocan (2012) Retrieved from www.globocan.iarc.fr.

2. Ajani JA, Winter K, Okawara GS, et al. (2006) Phase II trial of preoperative chemoradiation in patients with localized gastric adenocarcinoma (RTOG 9904): quality of combined modality therapy and pathologic response. J Clin Oncol 24(24):3953-3958

3. Allum WH, Stenning SP, Bancewicz J, Clark PI, Langley RE (2009) Long-term results of a randomized trial of surgery with or without preoperative chemotherapy in esophageal cancer. J Clin Oncol 27(30):5062-5067

4. Cunningham D, Allum WH, Stenning SP, et al. (2006) Perioperative chemotherapy versus surgery alone for resectable gastroesophageal cancer. N Engl J Med 355(1):11-20

5. Claassen YHM, Hartgrink HH, Dikken JL, et al. (2018) Surgical morbidity and mortality after neoadjuvant chemotherapy in the CRITICS gastric cancer trial. Eur J Surg Oncol 44(5):613-619

6. van Hagen P, Hulshof MC, van Lanschot JJ, et al. (2012) Preoperative chemoradiotherapy for esophageal or junctional cancer. N Engl J Med 366(22):2074-2084

7. Al-Batran SE, Hofheinz RD, Pauligk C, et al. (2016) Histopathological regression after neoadjuvant docetaxel, oxaliplatin, fluorouracil, and leucovorin versus epirubicin, cisplatin, and fluorouracil or capecitabine in patients with resectable gastric or gastro-oesophageal junction adenocarcinoma (FLOT4-AIO): results from the phase 2 part of a multicentre, open-label, randomised phase 2/3 trial. Lancet Oncol 17(12):1697-1708

8. Reynolds JV, Preston SR, O'Neill B, et al. (2017) ICORG 10-14: NEOadjuvant trial in adenocarcinoma of the oEsophagus and oesophagoGastric junction International Study (Neo-AEGIS). BMC Cancer 17(1):401

9. Miao ZF, Liu XY, Wang ZN, et al. (2018) Effect of neoadjuvant chemotherapy in patients with gastric cancer: a PRISMA-compliant systematic review and meta-analysis. BMC Cancer 18(1):118

10. Secrier M, Li X, de Silva N, et al. (2016) Mutational signatures in esophageal adenocarcinoma define etiologically distinct subgroups with therapeutic relevance. Nat Genet 48(10):1131-1141

11. Katona BW, Rustgi AK (2017) Gastric cancer genomics: advances and future directions. Cell Mol Gastroenterol Hepatol 3(2):211-217

12. Ichikawa H, Nagahashi M, Shimada $Y$, et al. (2017) Actionable gene-based classification toward precision medicine in gastric cancer. Genome Med 9(1):93

13. Lagergren P, Avery KN, Hughes R, et al. (2007) Health-related quality of life among patients cured by surgery for esophageal cancer. Cancer 110(3):686-693

14. Hayes T, Smyth E, Riddell A, Allum W (2017) Staging in esophageal and gastric cancers. Hematol Oncol Clin North Am 31(3):427-440

15. Findlay JM, Bradley KM, Maile EJ, et al. (2015) Pragmatic staging of oesophageal cancer using decision theory involving selective endoscopic ultrasonography, PET and laparoscopy. Br J Surg 102(12):1488-1499

16. Cancers NCCNGfEaEJ. National Comprehensive Cancer Network Guidelines for Esophageal and Esophagogastric Junction Cancers Retrieved from: https://www.nccn.org/professionals/physician_gls/ pdf/esophageal.pdf.

17. Foley KG, Christian A, Fielding P, Lewis WG, Roberts SA (2017) Accuracy of contemporary oesophageal cancer lymph node staging with radiological-pathological correlation. Clin Radiol 72(8):693el$693 \mathrm{e} 7$

18. Meyers BF, Downey RJ, Decker PA, et al. (2007) The utility of positron emission tomography in staging of potentially operable carcinoma of the thoracic esophagus: results of the American College of Surgeons Oncology Group Z0060 trial. J Thorac Cardiovasc Surg 133(3):738-745

19. Walker AJ, Spier BJ, Perlman SB, et al. (2011) Integrated PET/CT fusion imaging and endoscopic ultrasound in the pre-operative staging and evaluation of esophageal cancer. Mol Imaging Biol 13(1):166-171

20. Lordick F, Mariette C, Haustermans K, Obermannova R, Arnold D (2016) Oesophageal cancer: ESMO clinical practice guidelines for diagnosis, treatment and follow-up. Ann Oncol 27(suppl 5):v50 v57

21. Barber TW, Duong CP, Leong T, et al. (2012) 18F-FDG PET/CT has a high impact on patient management and provides powerful prognostic stratification in the primary staging of esophageal cancer: a prospective study with mature survival data. J Nucl Med 53(6):864-871

22. Goel R, Subramaniam RM, Wachsmann JW (2017) PET/computed tomography scanning and precision medicine: esophageal cancer. PET Clin 12(4):373-391

23. Seol KH, Lee JE (2014) PET/CT planning during chemoradiotherapy for esophageal cancer. Radiat Oncol J 32(1):31-42

24. le Grange F, Wickers S, Warry A, et al. (2015) Defining the target in cancer of the oesophagus: direct radiotherapy planning with fluorodeoxyglucose positron emission tomography-computed tomography. Clin Oncol (Royal College of Radiologists (Great Britain)) 27(3):160-167

25. Lee G, Hoseok I, Kim SJ, et al. (2014) Clinical implication of PET/ MR imaging in preoperative esophageal cancer staging: comparison with PET/CT, endoscopic ultrasonography, and CT. J Nucl Med 55(8):1242-1247

26. Tixier F, Le Rest CC, Hatt M, et al. (2011) Intratumor heterogeneity characterized by textural features on baseline 18F-FDG PET images predicts response to concomitant radiochemotherapy in esophageal cancer. J Nucl Med 52(3):369-378

27. Beukinga RJ, Hulshoff JB, van Dijk LV, et al. (2017) Predicting response to neoadjuvant chemoradiotherapy in esophageal cancer with textural features derived from pretreatment (18)F-FDG PET/ CT imaging. J Nucl Med 58(5):723-729

28. Nakajo M, Jinguji M, Nakabeppu Y, et al. (2017) Texture analysis of (18)F-FDG PET/CT to predict tumour response and prognosis of patients with esophageal cancer treated by chemoradiotherapy. Eur J Nucl Med Mol Imaging 44(2):206-214

29. Paul D, Su R, Romain M, et al. (2017) Feature selection for outcome prediction in oesophageal cancer using genetic algorithm and random forest classifier. Comput Med Imaging Graph 60:42-49

30. Foley KG, Hills RK, Berthon B, et al. (2018) Development and validation of a prognostic model incorporating texture analysis derived from standardised segmentation of PET in patients with oesophageal cancer. Eur Radiol 28(1):428-436

31. Tan S, Kligerman S, Chen W, et al. (2013) Spatial-temporal [(1)(8)F]FDG-PET features for predicting pathologic response of esophageal cancer to neoadjuvant chemoradiation therapy. Int $\mathbf{J}$ Radiat Oncol Biol Phys 85(5):1375-1382

32. van Rossum PS, Fried DV, Zhang L, et al. (2016) The incremental value of subjective and quantitative assessment of $18 \mathrm{~F}$-FDG PET for the prediction of pathologic complete response to preoperative chemoradiotherapy in esophageal cancer. J Nucl Med 57(5):691-700

33. Yip SS, Coroller TP, Sanford NN, et al. (2016) Use of registrationbased contour propagation in texture analysis for esophageal cancer pathologic response prediction. Phys Med Biol 61(2):906-922

34. Beukinga RJ, Hulshoff JB, Mul VEM, et al. (2018) Prediction of response to neoadjuvant chemotherapy and radiation therapy with baseline and restaging (18)F-FDG PET imaging biomarkers in patients with esophageal cancer. Radiology 287(3):983-992

35. Hou Z, Ren W, Li S, et al. (2017) Radiomic analysis in contrastenhanced CT: predict treatment response to chemoradiotherapy in esophageal carcinoma. Oncotarget 8(61):104444-104454

36. Ganeshan B, Skogen K, Pressney I, Coutroubis D, Miles K (2012) Tumour heterogeneity in oesophageal cancer assessed by CT texture analysis: preliminary evidence of an association with tumour metabolism, stage, and survival. Clin Radiol 67(2):157-164

37. Yip C, Landau D, Kozarski R, et al. (2014) Primary esophageal cancer: heterogeneity as potential prognostic biomarker in patients treated with definitive chemotherapy and radiation therapy. Radiology 270(1):141-148

38. Ba-Ssalamah A, Muin D, Schernthaner R, et al. (2013) Texturebased classification of different gastric tumors at contrast-enhanced CT. Eur J Radiol 82(10):e537-e543 
39. Ma Z, Fang M, Huang Y, et al. (2017) CT-based radiomics signature for differentiating Borrmann type IV gastric cancer from primary gastric lymphoma. Eur J Radiol 91:142-147

40. Liu S, Liu S, Ji C, et al. (2017) Application of CT texture analysis in predicting histopathological characteristics of gastric cancers. Eur Radiol 27(12):4951-4959

41. Yoon SH, Kim YH, Lee YJ, et al. (2016) Tumor heterogeneity in human epidermal growth factor receptor 2 (HER2)-positive advanced gastric cancer assessed by $\mathrm{CT}$ texture analysis: association with survival after trastuzumab treatment. PLloS ONE 11(8):e0161278

42. Giganti F, Antunes S, Salerno A, et al. (2017) Gastric cancer: texture analysis from multidetector computed tomography as a potential preoperative prognostic biomarker. Eur Radiol 27(5):1831-1839

43. Giganti F, Marra P, Ambrosi A, et al. (2017) Pre-treatment MDCT-based texture analysis for therapy response prediction in gastric cancer: Comparison with tumour regression grade at final histology. Eur J Radiol 90:129-137
44. Liu S, Zhang Y, Chen L, et al. (2017) Whole-lesion apparent diffusion coefficient histogram analysis: significance in $\mathrm{T}$ and $\mathrm{N}$ staging of gastric cancers. BMC Cancer 17(1):665

45. Liu S, Zhang Y, Xia J, et al. (2017) Predicting the nodal status in gastric cancers: the role of apparent diffusion coefficient histogram characteristic analysis. Magn Reson Imaging 42:144-151

46. Liu S, Zheng H, Zhang Y, et al. (2018) Whole-volume apparent diffusion coefficient-based entropy parameters for assessment of gastric cancer aggressiveness. J Magn Reson Imaging 47(1):168175

47. Zhang Y, Chen J, Liu S, et al. (2017) Assessment of histological differentiation in gastric cancers using whole-volume histogram analysis of apparent diffusion coefficient maps. J Magn Reson Imaging 45(2):440-449

48. Lambin P, Leijenaar RTH, Deist TM, et al. (2017) Radiomics: the bridge between medical imaging and personalized medicine. Nat Rev Clin Oncol 14(12):749-762 